\title{
Generalised semi-empirical correlation for heat transfer in channels of plate heat exchanger
}

\author{
Olga P. Arsenyeva ${ }^{\mathrm{b}, *}$, Leonid L. Tovazhnyanskyy ${ }^{\mathrm{b}}$, Petro O. Kapustenko ${ }^{\mathrm{a}}$, \\ Oleksiy V. Demirskiy ${ }^{\mathrm{a}}$ \\ a AO SPIVDRUZHNIST-T LLC, 2 Krasnoznamenny Per., 61002 Kharkiv, Ukraine \\ ${ }^{\mathrm{b}}$ Department of Integrated Technologies and Processes and Apparatuses, National Technical University "Kharkiv Polytechnic Institute", \\ 21 Frunze Str., 61002 Kharkiv, Ukraine
}

\section{H I G H L I G H T S}

- The analysis of turbulent flow in channels of PHEs.

- The analysis of the Prandtl number influence on heat transfer in PHE is performed.

- The semi-empirical model of turbulent heat transfer inside PHEs channels is proposed.

- The proposed model enables to design the PHEs for different duties.

\section{A R T I C L E I N F O}

\section{Article history:}

Received 18 February 2014

Received in revised form

13 April 2014

Accepted 15 April 2014

Available online 24 April 2014

\section{Keywords:}

Plate heat exchanger

Heat transfer

Heat and momentum transfer

Friction factor

Prandtl number

Reynolds number

\begin{abstract}
A B S T R A C T
The analogy of heat and momentum transfer in turbulent flow modified for channels of Plate Heat Exchanger (PHE) is proposed. The effects of channel geometry, flow velocity and fluid properties on heat transfer are accounted in the resulting equation, which permits the calculation of film heat transfer coefficients using the generalized correlation for friction factor at the main corrugated field of the interplate channel. The results of calculations are compared with data from experimental study. The good accuracy of film heat transfer coefficients prediction is shown. In the case when the corrugations direction is parallel to the flow direction, the calculations results are quite close to the predicted by the Equation published in the literature for straight pipes. The Prandtl number influence on heat transfer is discussed and semi-empirical Equation for its evaluation is proposed. The comparison with experimental data available in the literature confirmed the accuracy of the heat transfer prediction. The proposed Equation is recommended to be used for optimization of PHEs channels geometry for different conditions in the process industries. It can be employed also for optimizing PHEs heat exchange networks and also to determine PHEs heat transfer area targets when process integration methodology is employed.
\end{abstract}

(c) 2014 Elsevier Ltd. All rights reserved.

\section{Introduction}

Plate Heat Exchangers (PHEs) with intensified heat transfer are one of the most efficient types of modern heat transfer equipment. Their application in process industries, and especially in utilization of low grade heat, renewables and waste and biomass to energy technologies, as shown by Kilkovsky et al. [1], save space and construction material, enhance reliability and operability as compare to traditional shell and tube heat exchangers. The

\footnotetext{
* Corresponding author. Tel./fax: +380 577202278.

E-mail addresses: o.p.arsenyeva@gmail.com, olga.p.arsenyeva@gmail.com (O. P. Arsenyeva).
}

principles of operation of PHE and its design are well described in literature, as e.g. Ref. [2] and in Russian language [3]. PHE channels of intricate geometry are formed by plates produced by stamping from thin metal sheets. It induces high levels of turbulence leading to enhancement of heat transfer. The heat and hydraulic performance of the PHE is strongly influenced by the form of plate corrugations. PHE design must account for this factor [4]. The researches on heat transfer and pressure drop in PHE channels, published in literature, are presenting results in form of empirical correlations. The influence of flow velocity and fluid properties is usually accounted in such correlations by functions of Reynolds and Prandtl numbers. Such functions are of different forms, which are specific for studied channels, their geometries and the range of tested conditions. 


\begin{tabular}{|c|c|c|c|}
\hline \multicolumn{2}{|c|}{ Nomenclature } & $\begin{array}{l}\eta \\
\lambda\end{array}$ & $\begin{array}{l}\text { the dimensionless distance from the channel wall; } \\
\text { the heat conductivity, } \mathrm{W} /(\mathrm{m} \mathrm{K}) \text {; }\end{array}$ \\
\hline$b$ & height of corrugation, $\mathrm{m}$ & $\mu$ & the dynamic viscosity, cP; \\
\hline$c$ & the fluid heat capacity, $\mathrm{J} /(\mathrm{kg} \mathrm{K})$ & $\nu$ & the kinematic viscosity, $\mathrm{m}^{2} / \mathrm{s}$; \\
\hline$C^{*}$ & empirical coefficient & $\nu_{T}$ & the turbulent viscosity (momentum eddy diffusivity), \\
\hline$D_{e}$ & equivalent diameter of the channel, $\mathrm{m}$ & & $\mathrm{m}^{2} / \mathrm{s}$ \\
\hline$F_{x}$ & the surface area enlargement factor & $\rho$ & the fluid density, $\mathrm{kg} / \mathrm{m}^{3}$; \\
\hline$I$ & Integral in Eq. (4) & $\tau_{W}$ & shear stress at the wall, Pa; \\
\hline$R$ & the distance from the tube centre, $\mathrm{m}$ & $\omega$ & the relative velocity; \\
\hline$S$ & pitch of the corrugation, $\mathrm{m}$ & $\xi$ & the relative distance from the tube centre; \\
\hline & the local velocity, $\mathrm{m} / \mathrm{s}$ & $\psi$ & share of friction pressure losses in total loss of pressure \\
\hline & the average velocity, $\mathrm{m} / \mathrm{s}$ & & at the channel main corrugated field; \\
\hline & & $\zeta_{S}$ & the friction factor accounting for total pressure losses \\
\hline \multicolumn{2}{|c|}{ Numbers } & & in channel; \\
\hline \multicolumn{2}{|c|}{$N u=h \cdot D_{e} / \lambda$ the Nusselt number } & $\zeta_{\tau}$ & the friction factor for friction pressure losses \\
\hline \multicolumn{4}{|c|}{$\operatorname{Pr}=c \cdot \rho \cdot \nu / \lambda$ the Prandtl number } \\
\hline \multicolumn{2}{|c|}{$R e=w \cdot D_{e} \cdot \rho / \mu$ the Reynolds number } & \multicolumn{2}{|c|}{ Superscripts: } \\
\hline \multicolumn{4}{|c|}{ Greek letters } \\
\hline \multirow[t]{2}{*}{$\beta$} & the angle of corrugations inclination to flow direction, & Sub & \\
\hline & degrees; & $T$ & core of turbulent flow; \\
\hline$\beta_{M}$ & the proportionality coefficient; & $B$ & buffer sub-layer; \\
\hline$\chi$ & constant for turbulent flow in pipes; & $L$ & viscous sub-layer; \\
\hline$\varepsilon$ & the eddy diffusivities ratio for heat and momentum; & $W$ & the wall surface \\
\hline
\end{tabular}

The heat transfer and hydraulic performance of inter-plate channels affects the selection of the plate corrugation optimal geometry for PHE. The semi-empirical model of the link between heat and momentum transfer for turbulent flows in straight round pipes was firstly developed by Reynolds in 1874 and later modified by Prandtl in 1928 and Von Karman in 1939. Then it was utilized and developed by many researchers and has been proved useful in obtaining more accurate representation and physical background for turbulent heat transfer. In a number of practical cases it enabled to correlate experimental data and to extrapolate correlations for a wider range of their application. E.g. the correlation for heat transfer in straight pipes and channels presented by Gnielinski [5], based on the Prandtl analogy, has been proved accurate for turbulent and also for transitional flow regimes in a wide range of Prandtl numbers and is recommended by the authors of Perry's Chemical Engineers Handbook $\underline{[6]}$ for the use in practical applications.

To predict the heat transfer based on the data of friction factor in PHE channels Martin [7] have utilised the equation of the Leveque analogy, proposed initially for laminar flows. The same approach was employed later by Dović et al. [8] and it gave some reasonable accuracy in predicting data on heat transfer for a number of experimental data presented by different authors. The modification of Reynolds analogy for PHE channels was proposed by Tovazhnyansky and Kapustenko [9]. It showed a good agreement with data of their experimental study carried on the models of PHE channel's main corrugated field. Similar modification of the Reynolds analogy is proposed by Arsenyeva et al. [10] and is proved fairly accurate when comparing with the experimental data obtained from different studies of heat transfer inside the PHE channels. All these generalisation attempts use the fixed power at the Prandtl number in the correlating equation $(0.33$ in the Leveque equation and 0.4 fixed in the modified Reynolds analogy). According to the empirical correlations for different PHE channels published by different authors the power at the Prandtl number varies in fairly wide range, mostly from 0.3 up to 0.5 . The attempt to use the Gnielinski
Equation [5] for the PHE channels gives discrepancies with experimental results up to $300 \%$. The modification of the Von Karman analogy for PHE channels is proposed in paper [11], where the simplified assumption was made for turbulent viscosity distribution in buffer sub layer. The complicated resulting equation is rather cumbersome for the practical application of multi variant calculations in optimization problems.

In present paper more rigorous approach is presented. The results of the solution are approximated with the use of heat and momentum transfer Reynolds analogy and the equation which account the influence of the Prandtl number on heat transfer. The obtained equation enables to predict the film heat transfer coefficients for turbulent flows inside PHE channels in a wide range of the Reynolds and Prandtl numbers using the data for friction factor at the main corrugated field of the channel.

\section{The theoretical analysis}

Lyon [12] proposed one of the modifications of the Von Karman analogy inside pipes. The following Equation is derived there:

$\mathrm{Nu}^{-1}=2 \cdot \int_{0}^{1} \frac{\left(\int_{0}^{\xi} \omega \cdot \xi \cdot \mathrm{d} \xi\right)^{2}}{\left(1+\varepsilon \cdot \operatorname{Pr} \cdot \nu_{T} / \nu\right) \cdot \xi} \mathrm{d} \xi$

where $\xi=R / R_{0}$ is the relative distance from the centre of the pipe;

$\omega=w / W$ is the relative velocity and $w$ is the local velocity, $\mathrm{m} / \mathrm{s}$; $\varepsilon=\lambda_{T} /(c \rho) / \nu_{T}$ is the ratio of eddy diffusivities for the heat and momentum;

Eq. (1) can be used for any flow regime, with the correct estimation of the velocity, $\nu_{T}$ and $\varepsilon$ profiles, as it was shown by Lyon [12]. Kukuteladze [13] proposed the method to use Eq. (1) for the 
heat transfer estimation in turbulent flow inside pipes. In the present study the procedure and some assumptions of that method are adopted to turbulent flow inside PHE channels as it is shown below.

The flow in direction perpendicular to the wall of PHE channel is assumed to be divided into viscous sub-layer, buffer layer and turbulent main part of the stream. It is important to estimate the thickness of those layers in comparison to the channel's equivalent diameter $D_{e}$. Near the smooth surface the profile of velocity for uncompressible flows is closely linked to the shear stress on that wall $\tau_{W}$. Using the friction factor for the total hydraulic resistance of the PHE channel $\zeta_{S}$, it can be written:

$\tau_{W}=\frac{\zeta_{S} \cdot \psi}{F_{X}} \frac{\rho \cdot W^{2}}{8}=\zeta_{\tau} \cdot \frac{\rho \cdot W^{2}}{8}$

where $\psi$ is the relative share of pressure loss due to the friction in total pressure loss at the main corrugated field of the channel.

The correlations estimating $\zeta_{S}$ and $\psi$ for a range of corrugations geometries is proposed by Arsenyeva et al. [10]. It was shown in another paper [14] that for water stream inside PHE channels the effectively operating PHEs should have the values of shear stress on the wall in the range from $10 \mathrm{~Pa}$ to $100 \mathrm{~Pa}$ and even higher. Let's introduce dimensionless coordinate perpendicular to the wall $\eta$. Then the upper boundary of the buffer sub-layer can be judged using the following relation (in assumption that $\eta=\eta_{2}=30$ ):

$y=\eta \cdot \nu /\left(\tau_{W} / \rho\right)^{0.5}$

For the water properties at $50{ }^{\circ} \mathrm{C}$ and $\tau_{W}=10$ Pa the upper boundary of the buffer sub-layer is $y_{2}=0.17 \mathrm{~mm}$. It keeps diminishing to $y_{2}=0.05 \mathrm{~mm}$ for $\tau_{W}=100 \mathrm{~Pa}$. That constitutes from about $4 \%$ to $1 \%$ of equivalent diameter $D_{e}$. The thickness of the viscous sub-layer is about 5 times even smaller, as according to different published data its dimensionless upper boundary can be estimated as from $\eta_{1}=5$ to $\eta_{1}=7$. With such small thickness for the viscous sub-layer it can be concluded:

1. The variable $\xi$ is very close to unity, varying maximally from 0.95 to 1 .

2. Taking into account such dimensions the surface of the walls forming PHE channels can be regarded as smooth. To maintain metal quality during stamping, it can have curvature radius minimum $1-1.5 \mathrm{~mm}$ to be pressed from sheet metal with the thickness equal even to $0.4-0.5 \mathrm{~mm}$ without cracks.

3. Under the integration of the inner integral of Eq. (1) its biggest parts are outside of viscous sub-layer. It permits to assume $\omega=1$ for the whole flow region.

The right side of Eq. (1) can be regarded as a sum of integrals, what corresponds with the mentioned flow parts: the turbulent main part of the stream $I_{T}$, the buffer layer $I_{B}$ and viscous sub-layer $\mathrm{I}_{\mathrm{L}}$. These integrals are representing the effect of the specified region on the heat transfer. For $\mathrm{Pr}>1$ the main change of temperature is happening in buffer and viscous sub-layers, as can be judged from the literature (see e.g. Ref. [12]). The increasing of the Prandtl number shifts it closer to the wall, and the part of thermal resistance in the main stream is diminishing compare to thermal resistance of the whole flow. In such case the integral $\mathrm{I}_{\mathrm{T}}$ corresponding to the turbulent main part of the stream can be estimated under the assumption, that its value is approximately equal to that one in a flow core of the smooth tube with the diameter equal to the equivalent diameter of the channel and with the same shear stress on the wall. Assuming also that eddy diffusivities of heat and momentum are equal ( $\varepsilon=1$ ) and that there $\nu<\nu_{T}$, the Eq. (1) can be rewritten:

$$
\begin{aligned}
N u^{-1} \approx \frac{1}{2} \cdot\left(I_{T}+I_{B}+I_{L}\right)= & \frac{1}{2} \cdot\left(\int_{0}^{\xi_{2}} \frac{\xi^{3} d \xi}{\operatorname{Pr} \cdot \nu_{T} / \nu}+\int_{\xi_{2}}^{\xi_{1}} \frac{\xi^{3} d \xi}{1+\operatorname{Pr} \cdot \nu_{T} / \nu} \mathrm{d} \xi\right. \\
& \left.+\int_{\xi_{1}}^{1} \frac{d \xi}{1+\operatorname{Pr} \cdot \nu_{T} / \nu} \mathrm{d} \xi\right)
\end{aligned}
$$

At the central part of the tube $\left(0 \leq \xi \leq \xi_{2}\right)$ more detailed estimation than $\omega=1$ for the velocity profile is needed. Here the logarithmic velocity distribution can be utilized:

$w=w^{*}\left(C^{*}+\frac{1}{\chi} \cdot \ln \frac{v^{*} \cdot y}{\nu}\right)$

where $w^{*}=\left(\tau_{W} / \rho\right)^{1 / 2}, \mathrm{~m} / \mathrm{s} ; C^{*}$ is the constant;

$\chi$ is the constant estimated according to the experimental data for turbulent flows in pipes.

Assuming that the local shear stress is equal to the turbulent shear stress and is proportional to the distance from the centre $\tau=\tau_{W} \cdot \xi$, the turbulent viscosity can be estimated as:

$\nu_{T}=\frac{\tau_{T}}{\rho \cdot d w / d y}=\chi \cdot w^{*} \cdot y \cdot \xi$

In dimensionless form, accounting for Eqs. (2) and (3):

$\nu_{T} / \nu=\chi \cdot \operatorname{Re} \cdot \sqrt{\zeta_{\tau} / 32} \cdot(1-\xi) \cdot \xi$

The heat transfer in the main turbulent stream, after substituting Eq. (5) in first integral $I_{T}$ of Eq. (4) and integration:

$$
\begin{aligned}
I_{\mathrm{T}} & =\int_{0}^{\xi_{2}} \frac{\xi^{3} d \xi}{\operatorname{Pr} \cdot \nu_{T} / \nu} \\
& =\frac{\sqrt{32}}{\operatorname{Pr} \chi \operatorname{Re} \sqrt{\zeta_{\tau}}}\left[\ln \left(\frac{\operatorname{Re}}{\eta_{2}} \sqrt{\frac{\zeta_{\tau}}{32}}\right)-\frac{1}{2}\left(1-\frac{\eta_{2}}{\operatorname{Re}} \sqrt{\frac{32}{\zeta_{\tau}}}\right)^{2}-1+\frac{\eta_{2}}{\operatorname{Re}} \sqrt{\frac{32}{\zeta_{\tau}}}\right]
\end{aligned}
$$

In the buffer sub-layer of PHE channel $\left(\xi_{2} \leq \xi \leq \xi_{1}\right)$ the another logarithmic velocity profile correlation can be used, as established in experiments with smooth pipes and for turbulent flow near flat surfaces:

$w=w^{*}\left(C^{* \prime}+\frac{1}{\chi^{\prime}} \cdot \ln \frac{v^{*} \cdot y}{\nu}\right)$

Where $C^{* \prime}$ and $\chi^{\prime}$ are the empirical constants for the buffer sublayer.

Assuming the local shear stress $\tau$ in the buffer sub-layer as the sum of viscous and turbulent $\tau_{T}$ shear stresses and approximately equal to the wall shear stress, the turbulent viscosity can be determined as follows:

$\nu_{T}=\frac{\tau_{W}}{\rho \cdot d w / d y}-\nu \approx \chi^{\prime} \cdot w^{*} \cdot y-\nu$

In the dimensionless form, with accounting for Eqs. (2) and (3):

$\nu_{T} / \nu \approx \chi^{\prime} \cdot \operatorname{Re} \cdot \sqrt{\zeta_{\tau} / 32} \cdot(1-\xi)-1$ 
The heat transfer in the buffer sub-layer, after substituting Eq. (7) into the second integral $\mathrm{I}_{\mathrm{B}}$ of Eq. (4) and integration:

$$
\begin{aligned}
& I_{\mathrm{B}}=\int_{\xi_{2}}^{\xi_{1}} \frac{\xi^{3} d \xi}{1+\operatorname{Pr} \cdot \nu_{T} / \nu}=\int_{\xi_{2}}^{\xi_{1}} \frac{\xi^{3} d \xi}{\operatorname{Pr} \cdot \chi \prime \operatorname{Re} \sqrt{\frac{\zeta_{\tau}}{32}}(1-\xi)-(\operatorname{Pr}-1)}= \\
& =\frac{\sqrt{32}}{\operatorname{Pr} \chi^{\prime} \operatorname{Re} \sqrt{\zeta_{\tau}}}\left[Z^{3} \ln \left(\frac{1+\operatorname{Pr}\left(\chi^{\prime} \cdot \eta_{2}-1\right)}{1+\operatorname{Pr}\left(\chi^{\prime} \cdot \eta_{1}-1\right)}\right)+\frac{\eta_{2}-\eta_{1}}{\left.\operatorname{Re} \sqrt{\frac{\zeta_{\tau}}{32}}\left(1-Z+Z^{2}\right)\right]}\right.
\end{aligned}
$$

Where $Z=1-(\operatorname{Pr}-1) /\left(\operatorname{Pr} \chi^{\prime} \operatorname{Re} \sqrt{\zeta_{\tau} / 32}\right)$

In Eq. (4) the third integral $\mathrm{I}_{\mathrm{L}}$ characterises the heat transfer in the viscous sub layer. In this area and its parts closer to the wall surface is the biggest temperature gradient at the Prandtl numbers much higher than unity. It requires to account the influence of turbulent pulsations intruding from the outer layers. Following Kutateladze [13] it can be done by the relation:

$\nu_{T} / \nu \approx \beta_{M} \cdot \eta^{3}$

here $\beta_{M}$ is the proportionality coefficient, which value is estimated empirically as $\beta_{M} \approx 0.03$. According to [13] in integral $\mathrm{I}_{\mathrm{L}}$ for the heat transfer another empirical coefficient $\beta_{T}=\beta_{M} / \eta_{1}^{2}$ should be used, that gives the following:

$I_{L}=\int_{\xi_{1}}^{1} \frac{d \xi}{1+\operatorname{Pr} \cdot \nu_{T} / \nu}=\frac{1}{\operatorname{Re}} \sqrt{\frac{\xi_{\tau}}{32}} \int_{0}^{\eta_{1}} \frac{d \eta}{1+\operatorname{Pr} \cdot \beta_{T} \cdot \eta^{3}}$

The analytical expression for the integral of the function like $1 /$ $\left(a^{3}+x^{3}\right)$ is rather cumbersome and long. The value of this integral is estimated by the numerical integration on a computer using Mathcad software. For the turbulent velocity profile parameters and empirical coefficients in mentioned Equations the following was assumed: $\eta_{2}=30 ; \eta_{1}=6.8 ; \chi=0.37 ; \chi^{\prime}=0.2 ; \beta_{T}=\beta_{M} / \eta_{1}^{2}$. As a result of calculations the relation for the Nusselt number was obtained:

$N u=\frac{0.131 \cdot R_{\xi} \cdot \operatorname{Pr}}{\ln \left(\frac{R_{\xi}}{760}\right)-\frac{14450}{R_{\xi}^{2}}+\frac{340}{R_{\xi}}+B_{Z}+2.52 \operatorname{Pr} \cdot \varphi(\operatorname{Pr})}$

where
Accounting that for such low Prandtl numbers the share of the viscous sub layer in total resistance to the heat transfer becomes relatively smaller if compare to other parts of the stream this approximation can be used to save computing time.

\section{The comparison with experimental data and discussion of the results}

To check the limits of Eq. (10) application and its accuracy the results of calculations by it was compared with experimental data on heat transfer in the channels of different geometries. The experimental results for the models of PHE channels with different corrugation size and inclination angle are presented in paper [10] and were compared with the prediction by Eq. (10), as shown in Fig. 1. The values of the Prandtl numbers are taken from the data of experiments. The discrepancies between values of the Nusselt numbers calculated by Eq. (10) and experimental ones have not exceeded $15 \%$ with the mean square error equal to $6.5 \%$.

The empirical equation for the friction factor $\zeta_{S}$ in criss-cross flow PHE channels reported by Arsenyeva et al. [15] was used in these calculations. It enables to predict the friction factors at the main corrugated field of PHE channels formed by plates with inclined corrugations in a following range of corrugation parameters: the corrugations inclination angle $\beta$ is from $14^{\circ}$ to $72^{\circ}$; double height to pitch ratio $r$ is from 0.52 to 1.02 . The range of the Reynolds numbers is from 5 to 25,000. The difference between the sinusoidal and triangular (with rounded edges) shapes proved practically not significant for the friction factor. The equation is as follows:

$\zeta_{S}=8 \cdot\left[\left(\frac{12+p 2}{\operatorname{Re}}\right)^{12}+\frac{1}{(A+B)^{\frac{3}{2}}}\right]^{\frac{1}{12}}$

$A=\left[p 4 \cdot \ln \left(\frac{p 5}{\left(\frac{7 \cdot p 3}{\operatorname{Re}}\right)^{0.9}+0.27 \cdot 10^{-5}}\right)\right]^{16}$

$B=\left(\frac{37530 \cdot p 1}{\operatorname{Re}}\right)^{16}$

where $p 1, p 2, p 3, p 4, p 5$ are parameters defined by the channel's corrugation form.

$p 1=\exp (-0.15705 \cdot \beta)$;

$B_{Z}=1.85\left[Z^{3} \ln \left(\frac{1+5 \mathrm{Pr}}{1+0.36 \mathrm{Pr}}\right)+\frac{131.24}{R_{\xi}}\left(1-Z+Z^{2}\right)\right] ; \quad R_{\xi}=\operatorname{Re} \cdot \sqrt{\frac{\zeta_{S} \cdot \psi}{F_{X}}}$

$Z=1-\frac{(\operatorname{Pr}-1) \sqrt{32}}{0.2 \operatorname{PrR}_{\varsigma}}$

$\phi(\operatorname{Pr})=\frac{1}{\eta_{1}} \cdot \int_{0}^{\eta_{1}} \frac{d \eta}{1+\operatorname{Pr} \cdot \beta_{\mathrm{T}} \cdot \eta^{3}} \approx \frac{1.14}{\eta_{1}} \cdot \operatorname{Pr}^{-0.04} \cdot \operatorname{arctg}\left(\eta_{1} \cdot \sqrt[3]{\operatorname{Pr} \cdot \beta_{\mathrm{T}}}\right)$

The obtained approximation (11) of the integral $\mathrm{I}_{\mathrm{L}}$ solution deviates from the numerical solution not more than $\pm 2 \%$ for $3 \leq \operatorname{Pr} \leq 10^{4}$ and not more than $-8 \%$ for $\operatorname{Pr}$ as low as 0.69 .

$$
p 2=\frac{\pi \cdot \beta \cdot \gamma^{2}}{3}
$$




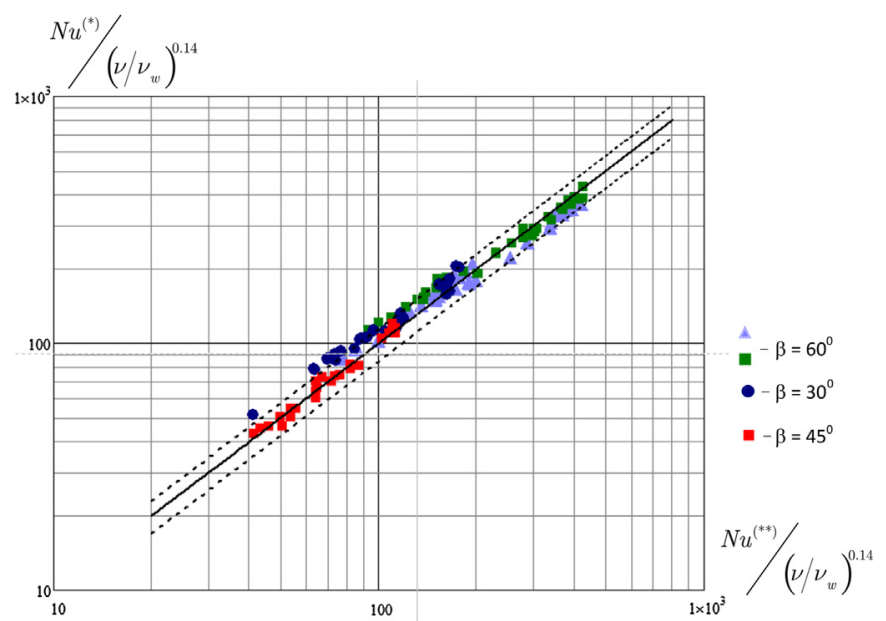

Fig. 1. Calculated by Eq. (10) Nusselt numbers $N u^{\text {calc }}$ (solid line) and experimental $N u^{\exp }$ : dashed lines corresponds to error $\pm 15 \%$.

$p 3=\exp \left(-\pi \cdot \frac{\beta}{180} \cdot \frac{1}{\gamma^{2}}\right)$

$p 4=\left(0.061+\left(0.69+\operatorname{tg}\left(\beta \cdot \frac{\pi}{180}\right)\right)^{-2.63}\right) \cdot\left(1+(1-\gamma) \cdot 0.9 \cdot \beta^{0.01}\right)$

$p 5=1+\frac{\beta}{10}$

The share of pressure losses due to friction $\psi$ in total pressure loss are determined by the following correlation presented in paper [10]:

$$
\begin{aligned}
\mathrm{A}_{1} & =380 /[\operatorname{tg}(\beta)]^{1.75} ; \quad \text { at } \quad \operatorname{Re}>\mathrm{A}_{1} \psi \\
& =\left(\operatorname{Re} / \mathrm{A}_{1}\right)^{-0.15 \cdot \sin (\beta)} ; \quad \text { at } \quad \operatorname{Re} \leq \mathrm{A}_{1} \psi=1
\end{aligned}
$$

The accuracy of Eq. (13) was checked and confirmed with CFD modelling of heat transfer in PHE channel presented by Stogiannis et al. [16].

The presented in Fig. 1 results of experiments were obtained for water as tested fluid in a limited range of the Prandtl numbers (from 1.9 to 7). The most of data available in the literature on heat transfer in PHE channels are reported as empirical correlations for the specific range of the Prandtl numbers, with no data on $\mathrm{Pr}$ in individual experimental runs. Arsenyeva et al. [10] has proposed the equation for film heat transfer coefficients calculation based on modification of the Reynolds analogy in the following form:

$N u=0.065 \cdot \operatorname{Re}^{6 / 7} \cdot\left(\psi \cdot \zeta_{\mathrm{s}} / \mathrm{F}_{\mathrm{X}}\right)^{3 / 7} \cdot \operatorname{Pr}^{\mathrm{c}} \cdot\left(\nu / \nu_{\mathrm{W}}\right)^{0.14}$

In this correlation the exponent at the Prandtl number is fixed to $c=0.4$. The comparisons with a number of published in the literature data was made by adjusting the results accounting for the value of $\operatorname{Pr}$ exponent in correlations and the range of $\operatorname{Pr}$ which was presented in those papers. The deviations not exceed $\pm 15 \%$. The comparison between calculations by Eqs. (10) and (14) at $\left(\nu / \nu_{W}\right)=1$ and $\mathrm{Pr}=1$ are presented in Fig. 2. The deviations not exceed 5\%. Therefore the conclusion can be made that in the region $0.7 \leq \operatorname{Pr} \leq 7$ the Eq. (10) predicts the influence of corrugations geometry on heat transfer practically with the same deviations as Eq. (14).

The accuracy of Eq. (10) under the accounting of the Prandtl number effect on heat transfer is also estimated by the comparison

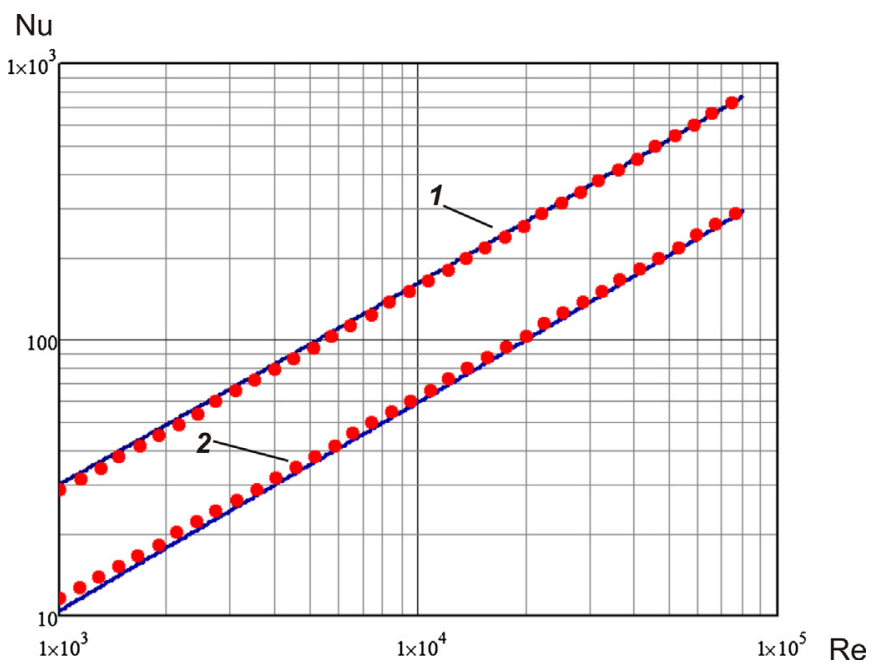

Fig. 2. Calculated by Eq. (10) Nusselt numbers $N u(10)$ (solid lines) and calculated by Eq. (14) $N u(4)$ (dotted lines) for $\nu / \nu_{W}=1$ and $\operatorname{Pr}=1: 1-\beta=65^{\circ} ; 2-\beta=30^{\circ}$.

with results obtained by calculations according to Gnielinski Equation [5] for the flows inside pipes and straight smooth channels:

$\mathrm{Nu}=\frac{\zeta_{\tau} \cdot \operatorname{Pr} \cdot(\operatorname{Re}-1000)}{8 \cdot\left[1+12.7 \cdot \sqrt{\zeta / 8 \cdot\left(\operatorname{Pr}^{2 / 3}-1\right)}\right]}$

Here $\zeta_{\tau}$ is the friction factor in a smooth tube determined by the correlation:

$\zeta_{\tau}=(0.79 \cdot \ln \operatorname{Re}-1.64)^{-2}$

For the analysis of Eq. (10) it was assumed that $\zeta_{s}=\zeta_{\tau} ; F_{x}=1$; $\psi=1$. The discrepancies of the results, which are presented in Fig. 3,

$$
N u /\left(\nu / \nu_{w}\right)^{0.14}
$$

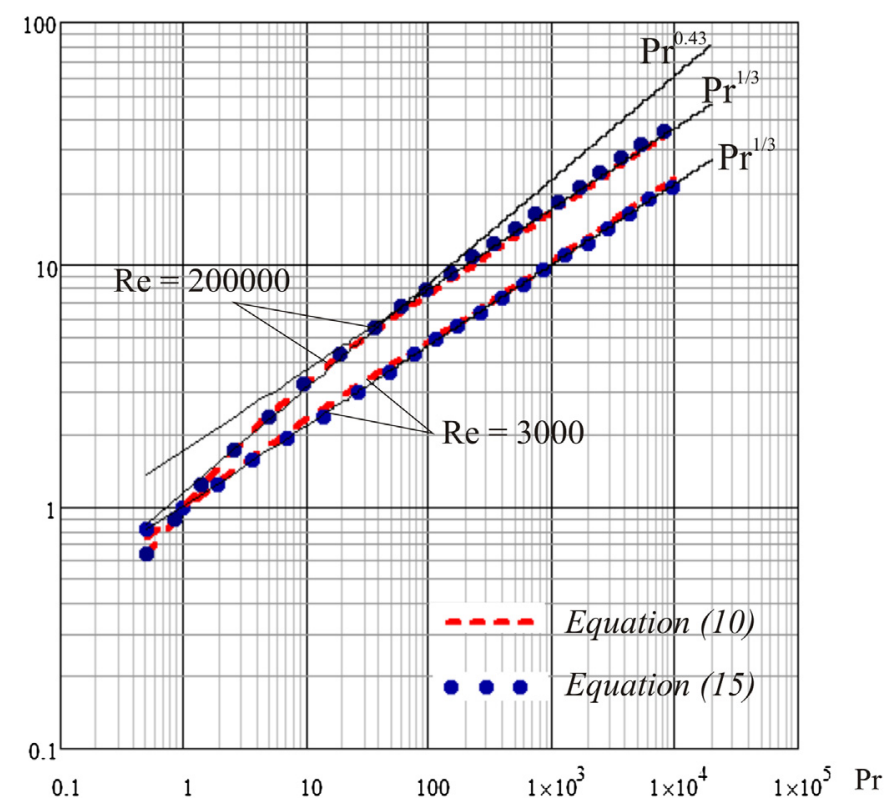

Fig. 3. The comparison of results by Eq. (10) with calculations by Eq. (15) and lines of correlations with fixed $\operatorname{Pr}$ exponents. 
not exceed 6\%. It is confirmed in the papers of Gnielinski [17] and by the authors of Perry's Chemical Engineers Handbook [6], that the accuracy of Eq. (15) is suitable for practical applications in wide range of the Prandtl numbers $(0.5-100,000)$. Therefore the good agreement of the results calculated by both Equations allows to recommend the Eq. (10) for the same range of Prandtl numbers not only for smooth tubes but also for PHE channels.

The effect on heat transfer of the Prandtl number is analysed with the graphs in Fig. 3. For the developed turbulent flow (the curves in Fig. 3 for $R e=200,000$ ) for $1.5 \leq P r \leq 12$ the curves can be approximated by using $\operatorname{Pr}$ exponent equal to 0.4 , as it is made in the Nusselt Equation (see Ref. [13]). In known Equation of Micheyev for this range of $\operatorname{Pr}$ the exponent 0.43 is used. When the Prandtl number is bigger than 20 , the exponent 0.33 can be applied, what is usually used for the heat transfer in laminar flows. This effect can be explained by the fact, that for higher Prandtl numbers the main part of the thermal resistance is shifting to the viscous sub-layer (3rd integral $\mathrm{I}_{\mathrm{L}}$ in Eq. (4)).

For lower Reynolds numbers (see the curves for $R e=3000$ in Fig. 3), which are corresponding to the transitional flow regime inside smooth tube, the exponent at the Prandtl number can be taken as 0.33 for all range of the Prandtl numbers. It can be explained by lower levels of turbulence at the main flow stream, causing smaller effect on flow in the regions with predominantly laminar heat transfer nature (the buffer and viscous sub-layers). Therefore the effect on heat transfer of the Prandtl number is determined not only by its value, but also by Reynolds number, which is characterizing the flow hydrodynamics in PHE channel. The accurate prediction of the heat transfer in a wide range of the Prandtl and the Reynolds numbers is not possible by introducing in correlation the Prandtl number as multiplier with some fixed exponent. The calculations must be made with Equations like (10) or (15), which account the flow conditions and the Reynolds number influence.

The Equation with the variable exponent at the Prandtl number for calculation of film heat transfer coefficients in PHE channels was used by Bogaert and Bölcs [18]. They have reported the results of the experimental study on heat transfer and pressure drop in a brazed PHE. The mineral oil NUTO H5, provided by ESSO, was used as a hot fluid. It was cooled by water during the experimental tests. In their calculations and presentation of results Bogaert and Bölcs [18] have used exponent at the Prandtl number presented by Eq. (17). It was extracted from the SWEP calculation procedure. Its accuracy is confirmed for all investigated range of the Reynolds $(1<\operatorname{Re}<1,000)$ and Prandtl $(2<\operatorname{Pr}<100)$ numbers.

$c_{1}(\operatorname{Pr})=\frac{1}{3} \cdot e^{\frac{6.4}{\operatorname{Pr}+30}}$

$$
c_{1}
$$

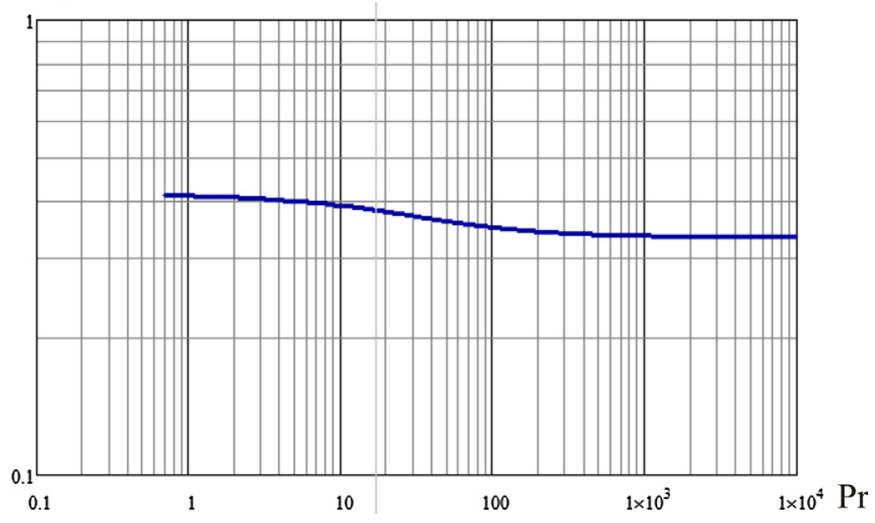

Fig. 4. The change of the Prandtl number exponent according to Eq. (17).
By this Equation the exponent at the Prandtl number is changing from 0.41 at $P r \approx 1$ to $\approx 0.333$ at $P r>800$ (see Fig. 4).

Bogaert and Bölcs [18] estimated that in their experiments the developed turbulent flow occurred at $R e>85$ and up to higher of investigated $R e=1,000$. The influence of $R e$ and $P r$ on heat transfer for corrugations parameters corresponding to experimental PHE from paper [18] is estimated by calculations carried out using the Eq. (10). The corrugations inclination angle is $\beta=68^{\circ}$, the corrugation aspect ratio $\gamma=0.6$ and the heat transfer area enlargement factor $F_{\mathrm{X}}=1.2$. For the range of the Reynolds numbers corresponding to the turbulent flow the influence of the Prandtl number calculated by Eq. (10) is in a good agreement with predicted using Prandtl power $\mathrm{c}_{1}$ determined by Eq. (17), as it is shown in Fig. 5 for $R e=200$. The discrepancies for $\operatorname{Pr}<100$ not exceed 4.2\%, but becoming bigger at higher $\operatorname{Pr}$ up to $9.9 \%$ at $\operatorname{Pr}=1,000$. The discrepancies at $R e=1000$ not exceed 5\% up to $P r=30$, becoming 9.5\% at $\operatorname{Pr}=100$ and $14.7 \%$ at $P r=1000$. Much bigger discrepancies are observed for $R e=10,000$, as shown in Fig. 6. The correction factor is introduced into Eq. (17) to account for the Reynolds number influence on the Prandtl number exponent, which becomes as follows:

$c_{2}(\operatorname{Re}, \operatorname{Pr})=\frac{1}{3} \cdot \frac{e^{\frac{6.4}{\mathrm{Pr}+30}}}{\left(1-0.012 \cdot \operatorname{Re}^{0.27}\right)}$

The comparison of the Prandtl influence on heat transfer calculated by Eq. (10) and predicted by introduction of the Prandtl exponent on Eq. (18) is presented in Fig. 6. The discrepancies not exceed $6 \%$ in the range of the Reynolds numbers from 100 to 20,000 and the Prandtl numbers from 0.7 to 1000 . The Prandtl number exponent calculated by Eq. (18) can be used in Eq. (14) for the prediction of film heat transfer coefficients in PHE channels. The comparison of the calculated by Eq. (14) results with data on heat transfer obtained by Bogaert and Bölcs [18] has demonstrated a good accuracy of prediction, as can be seen in Fig. 7. In the range of the Reynolds numbers $200>\operatorname{Re}>40$ the deviation from their correlations not exceed $2.1 \%$ at average $\operatorname{Pr}=50$ for oil and for $1000>\operatorname{Re}>230$ maximal discrepancy is $6.5 \%$ at $R e=1000$ at average $P r=4$ for water. According to Bogaert and Bölcs [18] this region of the Reynolds numbers corresponds to fully developed turbulent flow $(R e>85)$ and transitional quasi turbulent for the Reynolds number down to $40-50$. For the lower Reynolds numbers the flow becomes predominantly laminar and experimental values of film heat transfer coefficients much lower than predicted by Eq. (14).

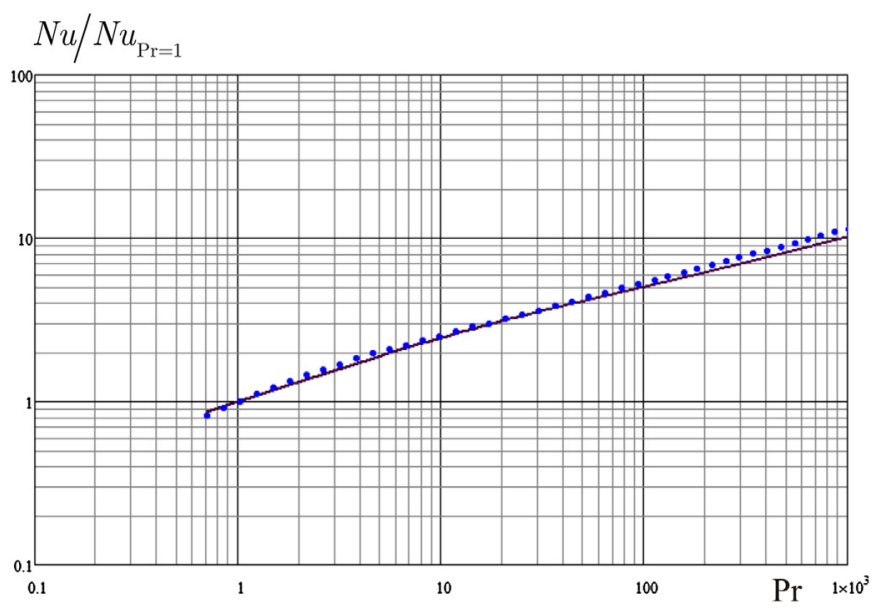

Fig. 5. The Prandtl number influence on heat transfer $N u / N u_{P r=1}$ at $R e=200$ : dotted line calculated by Eq. (10); solid line calculated at $\operatorname{Pr}^{\mathrm{c} 1}$. 


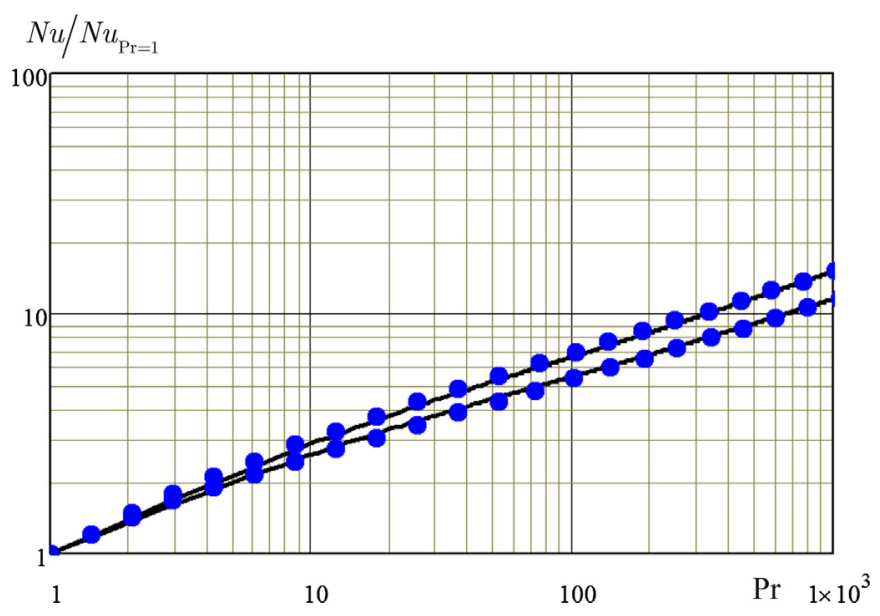

Fig. 6. The Prandtl number influence on heat transfer $\mathrm{Nu} / \mathrm{Nu}_{\mathrm{Pr}=1}$ at $\mathrm{Re}=200$ (lower lines) and $R e=10,000$ (upper lines): dotted lines calculated by Eq. (10); solid lines calculated at $\operatorname{Pr}^{\mathrm{c} 2}$ with Eq. (18).

The similar trend is observed when comparing calculation results with the experiments of Dovic et al. [8], as shown in Fig. 8. In that research were reported the results of tests with two models of PHE channels having corrugations with the angles at $\beta=65^{\circ}$ and $\beta=28^{\circ}$. The study was made with water and water glycerol solutions. The water data corresponds to Re numbers higher than 200. As the hydraulic diameter they used is $d_{\mathrm{h}}=2 b / F_{x}$, the values of $\mathrm{Nu}$ and $R e$ were corrected by $F_{x}=1.19$. The data for $N u$ at $R e>200$ were calculated at $\mathrm{Pr}=3.54$ (water $50^{\circ} \mathrm{C}$ ) to account for the difference in the exponent of $P r$. For the lower Reynolds numbers we considered average $\operatorname{Pr}=100$. The data were taken from a graph, so the accuracy is limited, but for $\operatorname{Re}>40$ at $\beta=65^{\circ}$ the model prediction (the upper solid line in Fig. 8) is fairly good. The error does not exceed $10 \%$. For the Reynolds numbers lower than 40 the experimental results become much lower than the calculated ones, same like results of the study made by Bogaert and Bölcs [18], see Fig. 7. For the channel with the smaller angle of corrugations $\beta=28^{\circ}$ the higher discrepancies are observed at $R e<80$. It is explained by the lower level of turbulence in PHE channels with smaller angle of corrugations, that

$$
N u / \operatorname{Pr}^{c_{1}}\left(\nu / \nu_{w}\right)^{0.14}
$$

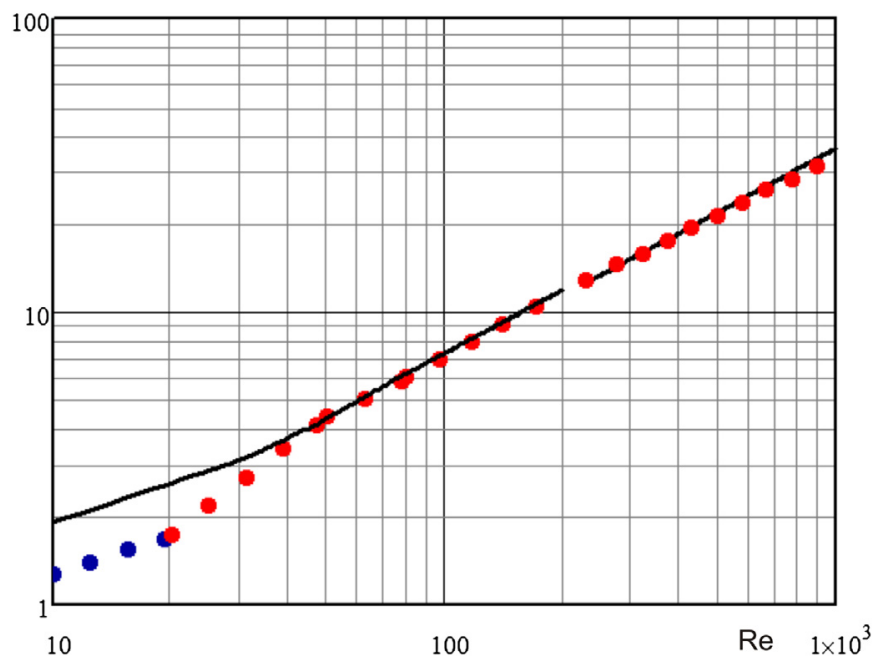

Fig. 7. The comparison of the calculation with experimental results of Bogaert and Bölcs [16]: solid lines are calculated by Eqs. (12), (14) and (18); dotted lines are calculated with correlations presented in paper [16].

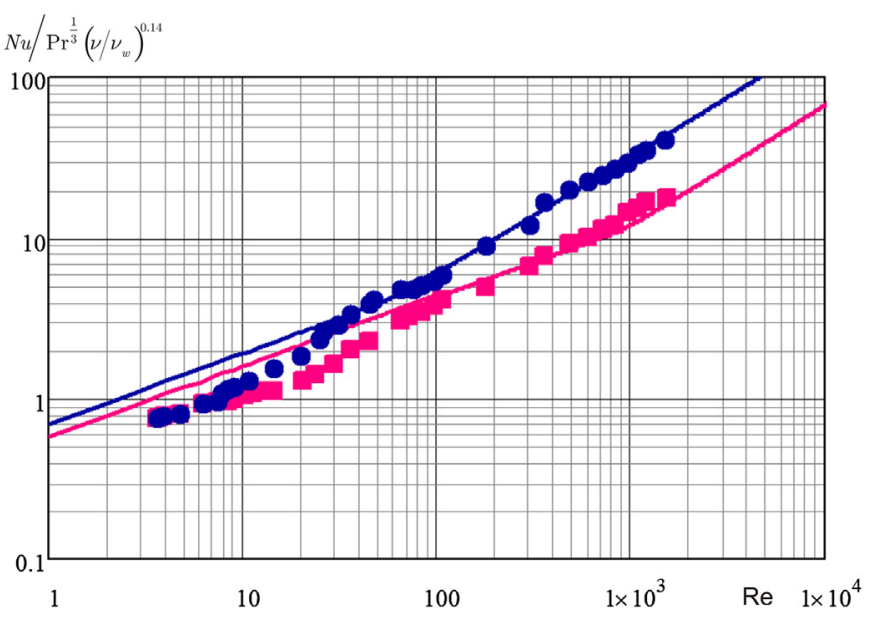

Fig. 8. The comparison of the calculation with experimental results of Dovic et al. [7]: solid lines are calculated by Eqs. (12), (14) and (18); dotted lines are calculated with correlations presented in paper of Dovic et al. (2009): round dots for $\beta=65^{\circ}$ and squires for $\beta=28^{\circ}$.

also leads to smaller heat transfer coefficients at the flow region with predominantly turbulent heat transfer $R e>80$. The predominantly laminar flow mechanism is taking place in such channels with the higher Reynolds numbers, up to 80 , compare to the channels with $\beta=65^{\circ}$ and $\beta=68^{\circ}$.

Another two studies of heat transfer in PHE channels, that have been made for the same channels in a wide range of the Prandtl numbers were reported by Muley, Manglik and Metwally [19] for $130<\operatorname{Pr}<290$ and $30<\operatorname{Re}<400$. Muley and Manglik [20] reported also data for the same channels at $2<\operatorname{Pr}<6$ and $1000<\operatorname{Re}<9000$.

The calculated by Eqs. (12), (14) and (18) results are in good agreement with the experimental data on heat transfer from paper [19], as it is shown in Fig. 9. The experimental data are presented by empirical correlations of that paper for average $\operatorname{Pr}=210$, as there are no data provided for the Prandtl numbers for individual experiments. As reported in paper [19], the accuracy of these empirical correlations is $\pm 10 \%$. The maximal discrepancy of calculated results with those by the correlations is not bigger than $12 \%$ that confirms the accuracy of the proposed calculation method. The experimental data for the channel with $\beta=30^{\circ}$ also show the deviation to lower values at $R e<80$, what is more clearly seen in the graph with experimental points in paper [19]. The good agreement

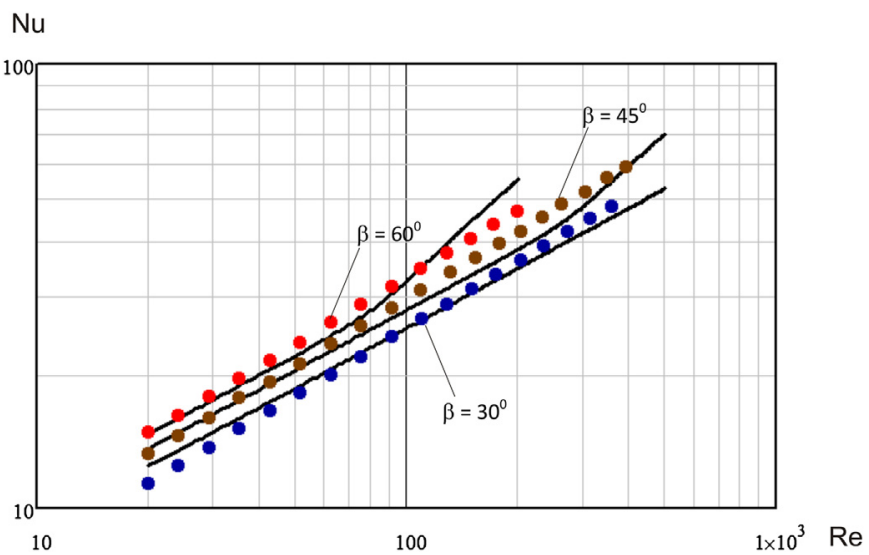

Fig. 9. The comparison of the calculation with experimental results of Muley, Manglik and Metwally [17]: solid lines are calculated by Eqs. (12), (14) and (18). 
of calculation by Eq. (14) with data from paper [20] was illustrated in paper [10].

The presented comparisons with experimental data of different researchers have confirmed the good accuracy of the proposed Equations for the prediction of film heat transfer coefficients in criss-cross channels of PHEs for flows with predominantly turbulent heat transfer mechanism. The application of Eq. (14) was confirmed earlier in paper [10] for developed turbulent flow in the range of the Reynolds numbers $100<\operatorname{Re}<25,000$ but for the limited range of the Prandtl numbers $0.7 \leq \operatorname{Pr} \leq 7$, what corresponds mostly to water heating or cooling. The range of the corrugations parameters was: $\beta$ from $14^{\circ}$ to $65^{\circ} ; \gamma$ from 0.5 to $1.5 ; F_{x}$ from 1.14 to 1.02 . The proposed above in the present paper function for accounting the Prandtl number influence on heat transfer, expressed by Eq. (18), allowed to extend the range of Eq. (14) application to the Prandtl numbers up to 1000 and corrugation angle up to $\beta=68^{\circ}$. The lower limit of the Reynolds numbers, in which the Eq. (14) keeps to be in good accuracy, depends of the corrugation angle $\beta$. For $\beta=65^{\circ}$ the Eq. (14) can be applied down to $R e=40$, but for the small angles $\beta=28^{\circ}$ only to $R e \approx 80$. At the lower Reynolds numbers the flow becomes predominantly laminar and advantages of heat transfer enhancement in PHE channels are not clear. Therefore in practice it can be recommended preferably not to use PHEs at such low Reynolds numbers in their channels.

\section{Conclusions}

The influence of the Reynolds and the Prandtl numbers on heat transfer in a wide range of their values cannot be accounted by simple power Equations with a constant as the Prandtl number exponent what is usually used in empirical correlations. The proposed semi-empirical model, which is based on a modification of Von Karman analogy for heat and momentum transfer, allows to predict the Prandtl number influence on heat transfer for flows in PHE channels. By approximation of the Eq. (10) solution the Eq. (18) is obtained, which gives the expression for the Prandtl number exponent depending on the Prandtl and Reynolds numbers.

The Eq. (18) can be used for determining the Prandtl number exponent in generalised correlation (14) for calculation of film heat transfer coefficients. It enables to estimate the heat transfer intensity in PHE channels based on correlation (12) for friction factor at the main corrugated field in such channels. The share $\psi$ of pressure drop due to the friction in total pressure drop should be calculated by Eq. (13). Such calculation procedure enables to obtain the film heat transfer coefficients with maximal error less than $15 \%$, as was demonstrated by the comparisons with experimental data on heat transfer published by a number of different researchers. These comparisons are presented in this paper and paper [10] published earlier. The introduction of Eq. (18) allowed to widen the range of applications of the proposed calculation procedure compare to the fixed Prandtl number exponent in correlation (14). It is estimated as follows: the Prandtl number $P r$ is from 0.7 to $10^{3}$; the Reynolds number $R e$ is from 80 to 25,000; the corrugation inclination angle $\beta$ is from $14^{\circ}$ to $68^{\circ}$; the corrugation aspect ratio $\gamma$ is from 0.5 to 1.02 ; the area enlargement factor $F_{X}$ is from 1.14 to 1.5. This range covers the most of the possible conditions for PHE applications in process industries, as well as feasible options of corrugations on the PHE plates. Together with Equations for estimation of local pressure drop in channel distribution zones and ports (as discussed in paper [21]) the presented Equations allow to develop the thermo hydraulic mathematical model of PHE. Such model can be used for optimization of PHEs channels geometry for different conditions in the process industries. It can be employed for developing software for optimisation of individual PHEs, as described in paper [22], and for optimizing PHEs heat exchange networks using approach discussed in paper [23].

\section{Acknowledgements}

Financial support from EC Project EFENIS (contract $\mathcal{N}$ ㅇ ENERFP7-296003) is sincerely acknowledged.

\section{References}

[1] B. Kilkovsky, P. Stehlik, Z. Jegla, L.L. Tovazhnyansky, O. Arsenyeva, P.O. Kapustenko, Heat exchangers for energy recovery in waste and biomass to energy technologies - I. Energy recovery from flue gas, Appl. Therm. Eng. $64(1-2)(2014) 213-223$.

[2] L. Wang, B. Sunden, R.M. Manglik, PHEs. Design, Applications and Performance, WIT Press, Southhampton, UK, 2007.

[3] L.L. Tovazshnyansky, P.O. Kapustenko, G.L. Khavin, O.P. Arsenyeva, PHEs in Industry, NTU KhPI, Kharkiv, Ukraine, 2004 in Russian.

[4] F.A.S. Mota, M.A.S.S. Ravagnani, E.P. Carvalho, Optimal design of plate heat exchangers, Appl. Therm. Eng. 63 (1) (2014) 33-39.

[5] V. Gnielinski, Neue Gleichungen für den Wärme- und den Stoffübergang in turbulent durchströmten Rohren und Kanälen, Forsch. im Ingenieurwessen 41 (1) (1975) 8-16.

[6] D.W. Green, R.H. Perry, Perry's Chemical Engineers Handbook, eighth ed., McGrow-Hill, New-York, USA, 2008.

[7] H. Martin, Theoretical approach to predict the performance of chevron-type plate heat exchangers, Chem. Eng. Process 35 (1996) 301-310.

[8] D. Dović, B. Palm, S. Švaić, Generalized correlations for predicting heat transfer and pressure drop in plate heat exchanger channels of arbitrary geometry, Int. J. Heat Mass Transf. 52 (19-20) (2009) 4553-4563.

[9] L.L. Tovazhnyansky, P.A. Kapustenko, Intensification of heat and mass transfer in channels of plate condensers, Chem. Eng. Commun. 31 (6) (1984) 351-366.

[10] O.P. Arsenyeva, L.L. Tovazhnyanskyy, P.O. Kapustenko, O.V. Demirskiy, Heat transfer and friction factor in criss-cross flow channels of plate-and-frame heat exchangers, Theor. Found. Chem. Eng. 46 (6) (2012) 634-641.

[11] O.P. Arsenyeva, L.L. Tovazhnyanskyy, P.O. Kapustenko, O.V. Demirskiy, The modified analogy of heat and momentum transfers for turbulent flows in channels of plate heat exchangers, Chem. Eng. Trans. 35 (2013) 487-492.

[12] R.N. Lyon, Liquid metal heat transfer coefficients, Chem. Eng. Prog. 47 (2) (1951) 75-79.

[13] S.S. Kutateladze, Fundamentals of Heat Exchange Theory, Atomizdat, Moscow, Russia, 1979.

[14] O.P. Arsenyeva, L.L. Tovazhnyanskyy, P.O. Kapustenko, O.V. Demirskiy, Accounting for thermal resistance of cooling water fouling in plate heat exchangers, Chem. Eng. Trans. 29 (2012) 1327-1332.

[15] O.P. Arsenyeva, L.L. Tovazhnyanskyy, P.O. Kapustenko, G.L. Khavin, The generalized correlation for friction factor in crisscross flow channels of plate heat exchangers, Chem. Eng. Trans. 25 (2011) 399-404.

[16] I.A. Stogiannis, S.V. Paras, O.P. Arsenyeva, P.O. Kapustenko, CFD modelling of hydrodynamics and heat transfer in channels of a PHE, Chem. Eng. Trans. 35 (2013) 1285-1290, http://dx.doi.org/10.3303/CET1335214.

[17] V. Gnielinski, Heat transfer coefficients for turbulent flow in concentric annular ducts, Heat Transf. Eng. 30 (6) (2009) 431-436.

[18] R. Bogaert, A. Bölcs, Global performance of a prototype brazed plate heat exchanger in a large Reynolds number range, Exp. Heat Transf. 8 (1995) 293311.

[19] A. Muley, R.M. Manglik, H.M. Metwally, Enhanced heat transfer characteristics of viscous liquid flows in a chevron plate heat exchanger, J. Heat Transf. 121 (1999) 1011-1017.

[20] A. Muley, R.M. Manglik, Experimental study of turbulent flow heat transfer and pressure drop in a plate heat exchanger with chevron plates, ASME J. Heat Transf. 121 (1999) 110-117.

[21] O.P. Arsenyeva, P.O. Kapustenko, L.L. Tovazhnyanskyy, G.L. Khavin, The influence of plate corrugations geometry on plate heat exchanger performance in specified process conditions, Energy 57 (2013) 201-207.

[22] O.P. Arsenyeva, L.L. Tovazhnyansky, P.O. Kapustenko, G.L. Khavin, Optimal design of plate-and-frame heat exchangers for efficient heat recovery in process industries, Energy 36 (8) (2011) 4588-4598.

[23] O.P. Arsenyeva, R. Smith, I. Bulatov, L.L. Tovazhnyanskyy, P.O. Kapustenko, G.L. Khavin, Estimation of enhanced heat transfer area targets in process industries, Comput. Aided Chem. Eng. 32 (2013) 355-360. 